\title{
Ecological Super-Spreaders Drive Host-Range Oscillations: Omicron and Risk-Space for Emerging infectious Disease
}

\author{
Walter A. Boeger ${ }^{1}$, Daniel R. Brooks ${ }^{2}$, Valeria Trivellone ${ }^{3}$, Salvatore Agosta ${ }^{4}$, and Eric \\ Hoberg $^{5}$ \\ ${ }^{1}$ Universidade Federal do Parana Setor de Ciencias Biologicas \\ ${ }^{2}$ Eötvös Loránd Research Network Centre for Ecological Research Institute of Evolution \\ 1121 Budapest Konkoly-Thege Miklós út 29-33 Hungary \\ ${ }^{3}$ University of Illinois Urbana-Champaign Prairie Research Institute \\ ${ }^{4}$ Stellenbosch Institute for Advanced Study \\ ${ }^{5}$ University of Wisconsin-Madison School of Veterinary Medicine
}

January 29, 2022

\begin{abstract}
Summary: The unusual genetic diversity of the Omicron strain has led to speculation about its origin. The mathematical modeling platform developed for the Stockholm Paradigm (SP) indicates strongly that it has retro-colonized humans from an unidentified animal reservoir originally infected by humans. The relationship between Omicron and all other SARS-CoV-2 variants indicates oscillations among hosts, a core part of the SP. Such oscillations result from the emergence of novel variants following colonization of new hosts, replenishing and expanding the risk space for disease emergence. The SP predicts that pathogens colonize new hosts using pre-existing capacities. Those events are thus predictable to a certain extent. Novel variants emerge after a colonization and are not predictable. This makes it imperative to take proactive measures for anticipating emerging infectious diseases (EID) and mitigating their impact. The SP suggests a policy protocol to accomplish this goal. This is the DAMA Protocol: comprising DOCUMENT to detect pathogens before they emerge in new places or colonize new hosts; ASSESS to determine risk; MONITOR to detect changes in pathogen populations that increase the risk of outbreaks; and $\mathrm{ACT}$ to prevent outbreaks when possible and minimize their impact when they occur.
\end{abstract}

\section{Hosted file}

manuscript-Lessons Omicron-TBED.docx available at https://authorea.com/users/391384/articles/ 554520-ecological-super-spreaders-drive-host-range-oscillations-omicron-and-risk-spacefor-emerging-infectious-disease 